Special Issue of the 6th International Congress \& Exhibition (APMAS2016), Maslak, Istanbul, Turkey, June 1-3, 2016

\title{
Composite Sponges for in Situ Alveolar Bone Regeneration Following Tooth Extraction
}

\author{
Z.A. GuClu ${ }^{a}$, A.P HurT ${ }^{b}$, L. Ohia ${ }^{b}$ And N.J. Coleman ${ }^{b, *}$ \\ ${ }^{a}$ Erciyes University, Department of Paediatric Dentistry, 38039 Kayseri, Turkey \\ ${ }^{b}$ University of Greenwich, Faculty of Engineering and Science, Chatham Maritime, Kent, ME4 4TB, UK \\ This research concerns the development of solvent-cast lyophilised composite sponges in the bioactive glass- \\ alginate-chitosan system for alveolar bone tissue maintenance following tooth extraction. Hydroxyapatite formed \\ on the surfaces of pure alginate, 50:50 alginate:chitosan blend and pure chitosan sponges blended with $10 \mathrm{wt} . \%$ \\ bioactive glass within 7 days of exposure to simulated body fluid, indicating that they possess the potential to \\ stimulate bone tissue formation. In the absence of bioactive glass, pure chitosan sponges also demonstrated in \\ vitro bioactivity, to a lesser extent; unlike pure alginate and 50:50 alginate:chitosan blend, which did not. All sam- \\ ples formed macroporous sponges whose biocompatibility with human osteosarcoma cells increased as a function \\ of chitosan-content. Polyelectrolyte complex formation between alginate and chitosan, and the incorporation of \\ bioactive glass were found to increase the swelling capacity of the sponges in SBF. The findings of this study de- \\ monstrate that, bioactive glass-chitosan sponges are the favoured candidates for alveolar bone tissue augmentation \\ as their rate of hydroxyapatite formation and biocompatibility are superior to those of the other samples.
}

DOI: 10.12693/APhysPolA.131.580

PACS/topics: 87.85.Lf

\section{Introduction}

Teeth may be lost or extracted due to poor oral hygiene, trauma and diseases such as cancer. In many cases the alveolar jaw bone is also compromised and requires augmentation prior to the placement of implanted prostheses. This research concerns the development of bioactive glass-polymer composite sponges for alveolar bone tissue regeneration, following tooth extraction.

Biodegradable, bioactive glasses, in the system $\mathrm{SiO}_{2}-$ $\mathrm{CaO}-\mathrm{P}_{2} \mathrm{O}_{5}-\mathrm{Na}_{2} \mathrm{O}$, are widely acknowledged to stimulate the regeneration of bone tissue and have been used, in particulate form, for the restoration of alveolar sockets $[1,2]$. These glass particles can be difficult to compact into the socket and are susceptible to "wash out" during placement. To address this problem, the incorporation of bioactive glass particles into biocompatible/biodegradable polymer sponges is proposed. These composite sponge materials could be readily cut into the desired shape and easily compacted into the vacant socket by the clinician.

Alginates are linear anionic carbohydrate co-polymers of $\beta$-D-mannuronate and $\alpha$-L-guluronate residues, derived from brown seaweed [3]. Sodium alginate is readily soluble in aqueous solutions and forms a gel in the presence of divalent cations, which cross-link the adjacent polymer chains to give a semi-rigid structure. Chitosan is the partially N-deacetylated derivative of chitin, obtained on an industrial scale from crustacean shells and some fungi, which is soluble under mildly acidic

*corresponding author; e-mail: n.coleman@gre.ac.uk conditions. It is a linear co-polymer of D-glucosamine and $\mathrm{N}$-acetylglucosamine, whose structure resembles that of bone extracellular matrix [4]. Alginate and chitosan are reported to be both biodegradable and biocompatible with bone tissue, with some evidence that chitosan is also bioactive with respect to bone tissue regeneration [3-5].

In this study, candidate composite sponges of mixtures of bioactive glass, alginate and chitosan were prepared by solvent-casting and freeze-drying (lyophilisation). The resulting materials were characterised by optical microscopy and Fourier transform infrared spectroscopy (FTIR). The bioactivity of the sponges was evaluated in vitro by monitoring the formation of hydroxyapatite on their surfaces after immersion in simulated body fluid (SBF). An indication of biocompatibility was also obtained using human MG63 osteosarcoma cells.

\section{Materials and methods}

All reagents were obtained from Sigma-Aldrich, UK, with the exception of the commercial bioactive glass, PerioGlas, which was supplied by NovaBone Products, Florida, USA.

Alginate:chitosan mixtures of mass ratio 10:0, 5:5, and 0:10 (viz. A10, A5 and A0) were prepared as $1 \%(\mathrm{w} / \mathrm{v}$ ) polymer solutions in $1 \%$ aqueous acetic acid. Similar mixtures were also prepared to which $10 \%$ bioactive glass, by total weight of polymer, were added (viz. A10-BG, A5-BG and A0-BG). Triplicate $5 \mathrm{~cm}^{3}$ aliquots of the solutions were frozen at $-18^{\circ} \mathrm{C}$ for $24 \mathrm{~h}$, then lyophilised for a further $24 \mathrm{~h}$ to produce initial sponges. Each of the sponges was then gelled for 30 minutes in $40 \mathrm{~cm}^{3}$ of $0.2 \mathrm{M}$ $\mathrm{CaCl}_{2(\mathrm{aq})}$, adjusted to $\mathrm{pH} 10$ with $\mathrm{NaOH}_{(\mathrm{aq})}$. These samples were washed with deionised water and again frozen and lyophilised to produce the final sponges, which were 
characterised by FTIR (Perkin Elmer RX1 spectrometer) and optical microscopy (Nikon Eclipse 90i microscope).

The viscosity of the A10, A5 and A0 solutions was measured as a function of applied shear stress (Bohlin Gemini 200 rheometer) in triplicate, using $4 \times 40 \mathrm{~mm}^{2}$ plates with a gap of $150 \mu \mathrm{m}$.

The in vitro bioactivity of the sponges was evaluated by immersion in SBF for 3 and 7 days, as described elsewhere [4]. Their swelling capacity at $72 \mathrm{~h}$ in SBF was evaluated using the following formula (Eq. 1), which compares the damp-dried mass (Wm) with the original dry mass $(\mathrm{Dm})$ to give an indication of the scaffold's ability to imbibe aqueous fluids [6].

$$
\text { Swelling capacity }(\%)=\frac{(\mathrm{Wm}-\mathrm{Dm})}{\mathrm{Dm}} \times 100 \text {. }
$$

In vitro biocompatibility of the sponges was assessed using human MG63 osteosarcoma cells, as previously described [7]. Briefly, $1 \times 1 \times 4 \mathrm{~mm}^{3}$ sections of sponge were placed in direct contact with the osteosarcoma cells at a concentration of $10^{6}$ cells $/ \mathrm{cm}^{3}$ in 96 -well plates for $72 \mathrm{~h}$. Cell viability was measured by MTT assay.

\section{Results and discussion}

\subsection{Rheology of the polymer solutions and polyelectrolyte complex formation}

Plots of viscosity as a function of applied shear stress for $1 \%(\mathrm{w} / \mathrm{v})$ solutions of alginate, 50:50 alginate:chitosan mixture and chitosan in 1\% acetic acid are given in Fig. 1.

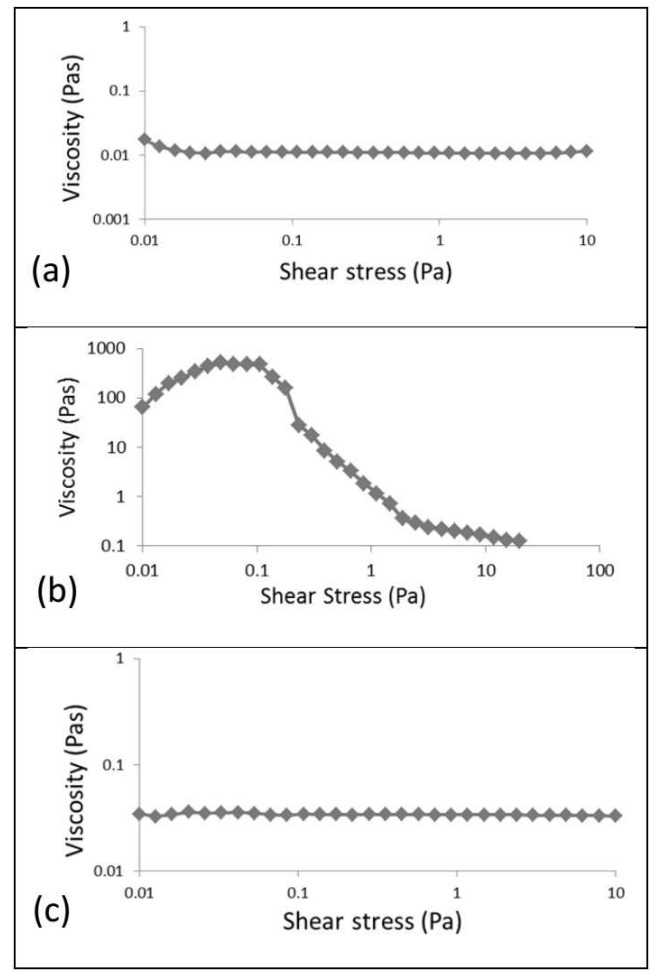

Fig. 1. Viscosity profiles of $1 \%(\mathrm{w} / \mathrm{v})$ solutions of (a) alginate, (b) 50:50 alginate:chitosan and (c) chitosan in $1 \%$ acetic acid.

The relatively low and invariant viscosity data for the individual alginate and chitosan solutions are typical of
Newtonian fluids, for which viscosity is independent of stress (Fig. 1a and c). Conversely, the comparatively high initial viscosity of the alginate:chitosan mixture, which rises and then diminishes as a function of applied stress, is indicative of the formation of a polyelectrolyte complex (PEC) between the two oppositely charged polymers in solution (Fig. 1b) [8].

The anionic alginate and cationic chitosan molecules form an electrostatically bonded polymer complex which is more bulky than the individual polymers and is characterised by increased viscosity at low shear stress. The decline in viscosity with increasing shear stress marks the point at which the applied shear force (onset at $\sim 0.3 \mathrm{~Pa}$ ) begins to disrupt the electrostatic forces of attraction within the PEC. This finding confirms the observations of other researchers who report that PEC formation between alginate and chitosan is driven by charge neutralisation and an increase in entropy, which accompanies the release of the counterions $[8,9]$.

\subsection{Optical microscopy of the polymer and composite sponges}

Optical micrographs of the individual polymer and composite sponges are shown in Fig. 2a-f. These images demonstrate that all polymer and composite blends form macroporous sponge-like materials with irregular interconnected porosity, the density of which increases as a function of chitosan-content. Interconnected macroporosity with pores in the observed range $(\sim 20-100 \mu \mathrm{m})$ are optimally sized for bone cell ingrowth and the diffusion of nutrients and metabolites [10]. The addition of bioactive glass is seen to increase the density of the sponges and also to reduce the pore size, without diminishing their potential functional capabilities as in situ tissue scaffolds.
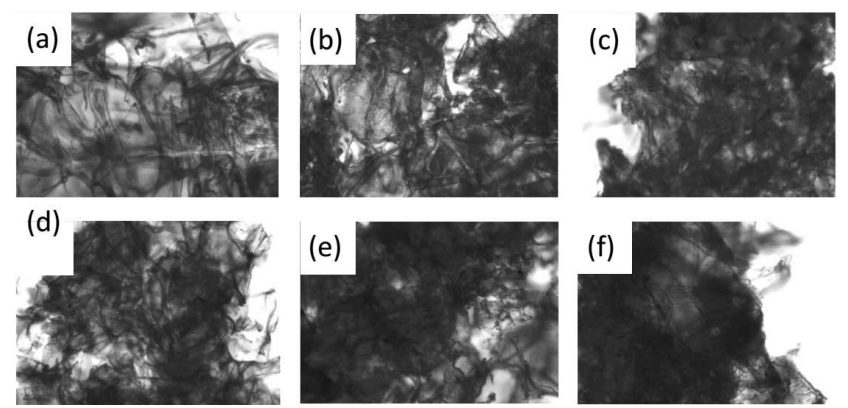

Fig. 2. Optical micrographs $(\times 10)$ of (a) A10, (b) A5, (c) A0, (d) A10-BG, (e) A5-BG and (f) A0-BG samples.

\subsection{In vitro bioactivity of composite sponges}

FTIR spectra of the polymer sponges (A10, A5 and $\mathrm{A} 0)$ prior to and following immersion in SBF for 3 and 7 days are shown in Fig. 3. The broad signal at $3450 \mathrm{~cm}^{-1}$ in the alginate spectrum arises from $\mathrm{O}-\mathrm{H}$ stretching modes (Fig. 3a) [9]. Signals common to all pure and blended polymer samples are the aliphatic $\mathrm{C}-\mathrm{H}$ stretching vibrations which occur at $2965-2955 \mathrm{~cm}^{-1}$, and $\mathrm{C}-\mathrm{H}$ 
bending modes which give rise to the bands at 1430 $1420 \mathrm{~cm}^{-1}[4,9]$. Carbonyl $\mathrm{C}=\mathrm{O}$ stretching modes appear at $\sim 1650 \mathrm{~cm}^{-1}$, various $\mathrm{C}-\mathrm{O}-\mathrm{C}$ stretching frequencies occur in the range $1160-1060 \mathrm{~cm}^{-1}$ and the band at $\sim 1295 \mathrm{~cm}^{-1}$ is attributed to $\mathrm{C}-\mathrm{O}-\mathrm{H}$ stretching vibrations in all polymer samples. In the FTIR spectrum of chitosan (Fig. 3c), the broad signal at $\sim 3460 \mathrm{~cm}^{-1}$ is attributed to $\mathrm{N}-\mathrm{H}$ and $\mathrm{O}-\mathrm{H}$ stretching modes, which overlap in this region; and the bands at 1650 and $1570 \mathrm{~cm}^{-1}$ arise from amide $\mathrm{I} \mathrm{C}=\mathrm{O}$ stretching and amide II $\mathrm{N}-\mathrm{H}$ bending vibrations, respectively [4]. A peak shift to lower wavenumber is seen in the amide I band of chitosan in the A5 sponge, indicating an interaction between the two polymers, which confirms polyelectrolyte complex formation.
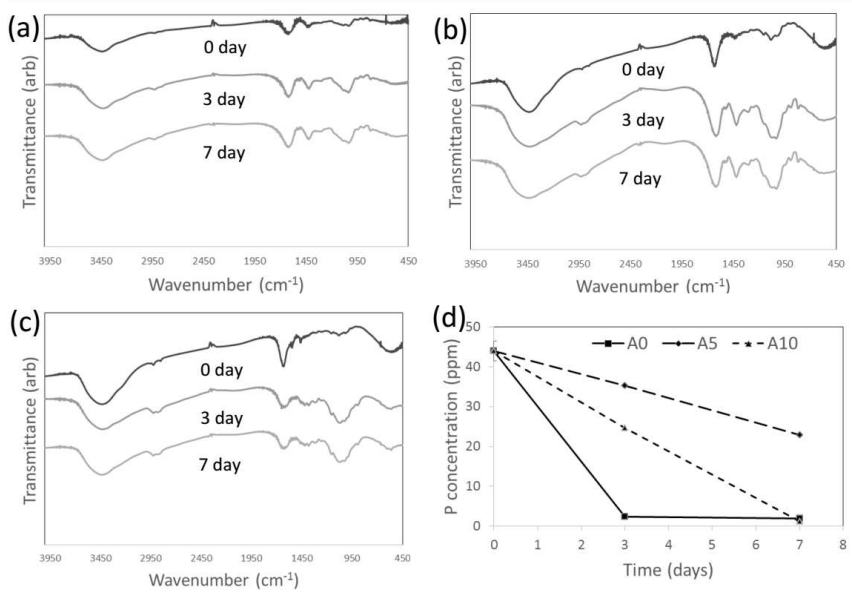

Fig. 3. FTIR spectra of (a) A10, (b) A5, (c) A0 and (d) corresponding SBF phosphate concentrations as functions of time.
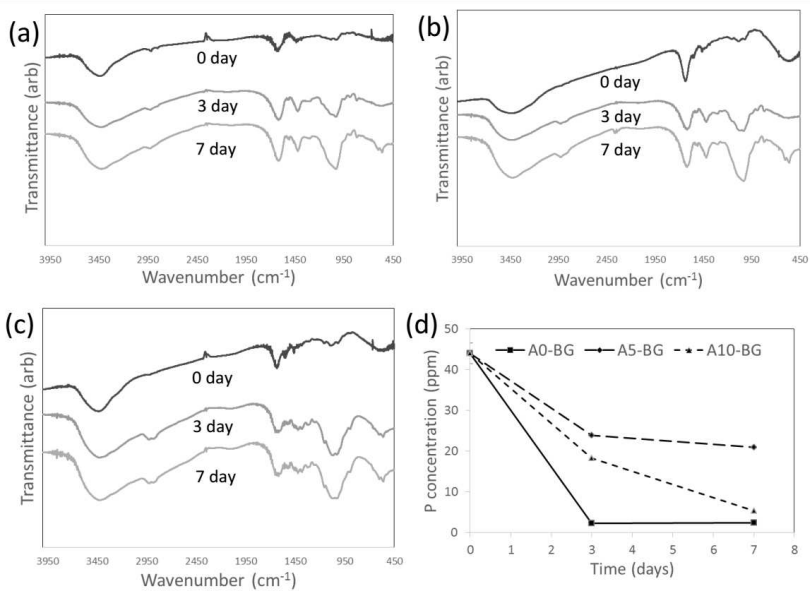

Fig. 4. FTIR spectra of (a) A10-BG, (b) A5-BG, (c) A0-BG and (d) corresponding SBF phosphate concentrations as functions of time.

FTIR spectra of the composite bioactive glass-polymer sponges prior to and following immersion in $\mathrm{SBF}$ for 3 and 7 days are shown in Fig. 4. Bands arising from $\mathrm{Si}_{-}$ $\mathrm{O}-\mathrm{Si}$ stretching modes of the bioactive glass contribute to the signals that appear in the $1000-900 \mathrm{~cm}^{-1}$ region of the spectra [11].

The in vitro formation of a surface layer of substituted hydroxyapatite, $(\mathrm{Ca}, \mathrm{Mg}, \mathrm{Na})_{10}\left(\mathrm{PO}_{4}, \mathrm{CO}_{3}, \mathrm{Cl}\right)_{6}(\mathrm{OH})_{2}$, (HA) on the surface of a material placed in $\mathrm{SBF}$ solution provides an indication of its bioactivity (i.e. the ability of the material to bond with living bone tissue). Characteristic phosphate $\mathrm{P}-\mathrm{O}$ bending modes of crystalline HA are observed at $570-610 \mathrm{~cm}^{-1}$ in the FTIR spectra of A10-BG and A5-BG after 7 days' residence in SBF (Fig. 4a and b). The concomitant removal of phosphate ions from the SBF is plotted in Fig. 4d. In comparison, the kinetics of $\mathrm{HA}$ precipitation on sample A0-BG is more rapid than those of the alginate-bearing composite sponges, as HA is seen to form within three days on this material.

In the absence of bioactive glass, no $\mathrm{HA}$ is detected on the surfaces of the A10 or A5 polymer sponges within 7 days (Fig. 3a and b); although, the SBF concentrations of the supernatant SBF solutions do steadily decrease during this time (Fig. 3d), indicating that phosphate ions are being adsorbed onto the polymer system. In contrast, the characteristic phosphate $\mathrm{P}-\mathrm{O}$ bending modes of $\mathrm{HA}$ are observed in the spectrum of the pure chitosan sponge (A0) after 7 days of exposure to SBF (Fig. 3c).

These findings indicate that in vitro bioactivity increases as a function of chitosan-content and that this property is markedly enhanced by the presence of $10 \mathrm{wt} . \%$ of bioactive glass.

\subsection{In vitro swelling capacity and cytocompatibility of composite sponges}

The initial swelling capacities of the polymer and composite sponges following immersion in SBF for 3 days are presented in Fig. 5a. The initial swelling capacity of a tissue scaffold is an important parameter, as the scaffold is required to absorb body fluid which promotes tissue ingrowth and facilitates the transmission of nutrients and metabolites [12].

Chitosan and alginate are both known to swell in aqueous solutions as water interacts with the polar functional groups of the polymers [12]. In this study, the swelling capacity of the polymer sponges (A10, A5 and A0) is principally influenced by the formation of the polyelectrolyte complex, which enhances swelling capacity (Fig. 5a). The incorporation of bioactive glass has no impact on the swelling capacity of the PEC sponge; whereas, it significantly enhances the fluid uptake of the alginate (A10$\mathrm{BG}$ ) and chitosan (A0-BG) composites.

The in vitro cell viability (i.e. biocompatibility) data for human osteosarcoma cells, cultured in contact with the polymer and composite sponges (relative to the control, which consisted of cells and media only) are given in Fig. 5b. These data indicate that biocompatibility increases with increasing chitosan-content. In this respect, both A0 and A0-BG sponges were found to enhance cell viability relative to the control, with the greatest increase noted for the bioactive glass-bearing composite. This finding differs from that of $\mathrm{Li}$ et al. [12] who report that 


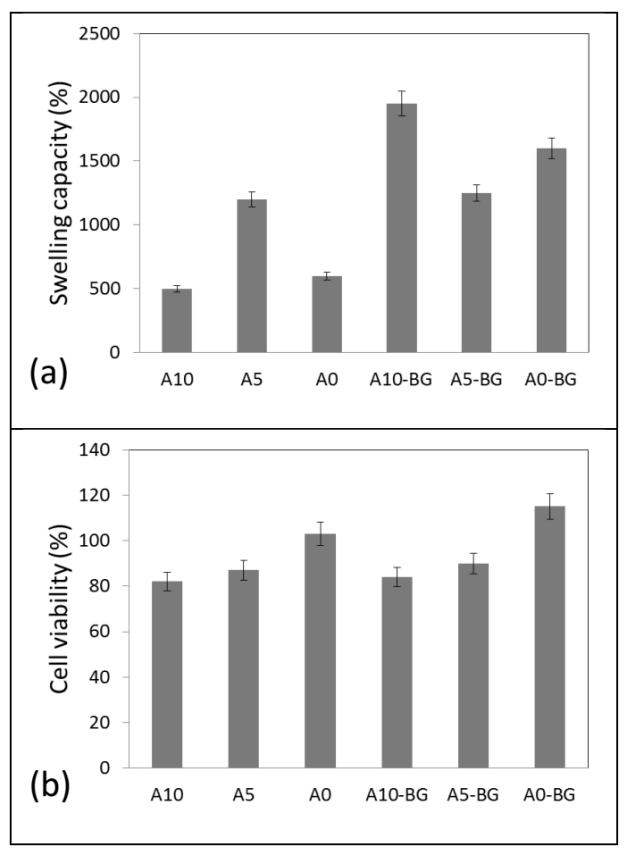

Fig. 5. In vitro (a) swelling capacities and (b) cytocompatibilities of polymer and composite sponges.

human osteosarcoma cells better proliferate on alginatechitosan blends than on pure chitosan. Presumably this disparity arises from the different cell culture environments and/or different textures and pore structures of the sponges prepared in the two studies.

\section{Conclusions}

This research concerns the development of bioactive glass-polymer composite sponges for alveolar bone tissue regeneration, following tooth extraction. Macroporous alginate:chitosan sponges of mass ratio 10:0, 5:5, and 0:10 were prepared by solvent-casting and lyophilisation with and without the addition of $10 \mathrm{wt} . \%$ of bioactive glass. The in vitro biocompatibility of all sponges was confirmed using human osteosarcoma cells, and cell viability was found to increase with increasing chitosancontent. In addition, the in vitro bioactivity of pure chitosan sponges and all composites blended with bioactive glass was verified by the formation of hydroxyapatite on their surfaces in simulated body fluid within seven days. Polyelectrolyte complex formation between alginate and chitosan, and the incorporation of bioactive glass were found to increase the swelling capacity of the sponges in SBF. The relatively rapid formation of hydroxyapatite and superior cytocompatibility of the bioactive glasschitosan composite indicates that this is potentially the most favourable candidate material for alveolar bone maintenance.

\section{References}

[1] J.R. Jones, Acta Biomaterialia 9, 4457 (2013).

[2] R. Margonar, T.P. Queiroz, E.R. Luvizuto, E. Marcantonio, R.C.C. Lia, M. Holzhausen, E. Marcantonio-Junior, J. Craniofacial Surg. 23, e220 (2012).

[3] J. Venkatesan, I. Bhatnagar, P. Manivasagan, K.H. Kang, S.K. Kim, Int. J. Biol. Macromol. 72, 269 (2015).

[4] A.P. Hurt, G. Getti, N.J. Coleman, Int. J. Biol. Macromol. 64, 11 (2014).

[5] M. Prabaharan, in: Polysaccharides, Eds. K.G. Ramawat, J.-M. Mérillon, Springer International Publishing, Switzerland, 2015, p. 1609.

[6] S. Saravanan, S. Nethala, S. Pattnaik, A. Tripathi, A. Moorthi, N. Selvamurugan, Int. J. Biol. Macromol. 49, 188 (2011).

[7] A.P. Hurt, A.K. Kotha, V. Trivedi, N.J. Coleman, Polimeros 25, 311 (2015).

[8] H. Vogt Sether, H.K. Holme, G. Maurstad, O. Smidsrod, B.T. Stokke, Carbohydr. Polym. 74, 813 (2008).

[9] M.G. Sankalia, R.C. Mashru, J.M. Sankalia, V.B. Sutariya, Eur. J. Pharm. Biopharm. 65, 215 (2007).

[10] J. Han, Z. Zhou, R. Yin, D. Yang, J. Nie, Int. J. Biol. Macromol. 46, 199 (2010).

[11] A.M. Deliormanli, Ceram. Int. 41, 417 (2015).

[12] Z. Li, H.R. Ramay, K.D. Hauch, D. Xiao, M. Zhang, Biomaterials 26, 3919 (2005). 